\title{
ESTRUTURA DE PESQUISA EM REDE: O CASO DA REDE NACIONAL DE PESQUISAS CLÍNICAS EM HOSPITAIS DE ENSINO
}

\author{
Marge Tenório \\ Ministério da Saúde do Brasil \\ Jesús Pascual Mena-Chalco \\ Universidade Federal do ABC \\ Guilherme Arantes Mello \\ Escola Paulista de Medicina - Universidade Federal de São Paulo - UNIFESP \\ Ana Luiza D’Ávila Viana \\ Faculdade de Medicina da Universidade de São Paulo
}

\begin{abstract}
Resumo
A pesquisa clínica no Brasil precisa rever a parceria entre a indústria farmacêutica, a academia científica e o governo. A Rede Nacional de Pesquisa Clínica em Hospitais Docentes (RNPC) foi criada em 2005, com o objetivo de estabelecer uma rede de ensaios brasileiros. E com o objetivo de destacar as características da RNPC que dificultam ou promovem esforços de pesquisa clínica bem-sucedidos, este estudo descritivo exploratório foi desenvolvido com uma abordagem de estudo de caso qualitativo. Na pesquisa, o software Gephi foi usado para visualizar a rede usando o algoritmo de layout Force Atlas 2. Os resultados bem-sucedidos estão relacionados ao treinamento de profissionais, a promoção de pesquisa com foco no SUS em intercâmbio acadêmico e técnico entre as instituições.
\end{abstract}

Palavras-chave: Estudos clínicos. Rede brasileira de pesquisas. SUS.

\section{NETWORK RESEARCH STRUCTURE: THE CASE OF THE NATIONAL NETWORK OF CLINICAL RESEARCH IN SCHOOL HOSPITALS}

\begin{abstract}
Clinical research in Brazil needs to review the partnership between the pharmaceutical industry, the scientific academy and the government. The National Network of Clinical Research in Teaching Hospitals (RNPC) was created in 2005, aiming to establish a Brazilian network of trials. And aiming to highlight the characteristics of RNPC that hinder or promote successful clinical research efforts, this exploratory descriptive study was developed with a qualitative-case study approach. In the research, the Gephi software was used to visualize the network using the Force Atlas 2 layout algorithm. The successful results are related to the training of professionals, the promotion of research with a focus on SUS in academic and technical interchange between the institutions.
\end{abstract}

Keyword: Clinical research. Brazilian network of research. SUS. 


\section{INTRODUÇÃO}

Entre os setores produtivos de Pesquisa e Desenvolvimento (P\&D) ${ }^{1}$, o desenvolvimento das fases clínicas de um medicamento, devidamente precedidas das pesquisas básicas e préclínicas, constitui um dos mais longos, onerosos e elaborados processos, motivando, desse modo, os arranjos colaborativos com a indústria farmacêutica como a forma mais viável para execução desses estudos ${ }^{2}$. De acordo com o Banco Nacional de Desenvolvimento (BNDES), o lançamento de um único produto novo pode comprometer até mais de dez anos de pesquisas, demandando investimentos superiores a US\$ 1 bilhão. De cada conjunto de cem mil novos compostos estudados, estima-se que apenas 250 dispõem de requisitos suficientes para prosseguir nos testes pré-clínicos (estudos em animais). Destes, meramente cinco iniciam as fases de ensaios clínicos em humanos por apresentarem segurança, chegando ao mercado $^{1}$, por fim tão somente um novo e determinado medicamento.

Historicamente diversos e variados protocolos de pesquisa clínica vinham sendo desenvolvidos pela indústria farmacêutica internacional no Brasil, por meio da compra de serviços, porém sem o efetivo comprometimento com as demandas da saúde pública do país ${ }^{3,4}$. A participação da comunidade científica brasileira, nesse processo, limitava-se à coleta de dados segundo os protocolos de pesquisas pré-estabelecidos. Em geral, a análise e a posse dos dados adquiridos nos estudos, pertenciam aos parceiros estrangeiros e das Contract Research Organization (CRO). Justamente por conta dessa detenção, não está longe da verdade a afirmação de que a pesquisa clínica configura a única modalidade de pesquisa realizada no Brasil, em que a maior parte das decisões é tomada no exterior ${ }^{2}$, não sendo relevante, portanto, a manutenção dessa prática para os grupos de pesquisas, instituições e pacientes envolvidos, nem para o país enquanto promotor de Saúde e de Ciência, Tecnologia e Inovação. ${ }^{1,3,4}$.

Com a publicação da Resolução 196 de $1996^{5}$, posteriormente revogada pela Resolução 466 de $2012^{6}$, a qual regulamenta as diretrizes e normas de pesquisas envolvendo seres humanos, as atividades em pesquisa clínica foram significativamente facilitadas e ampliadas. O reconhecimento da capacitação brasileira no contexto da pesquisa clínica internacional tem favorecido as parcerias multicêntricas e proporcionado a profissionalização na área ${ }^{4}$. Atualmente há 1.735 pesquisadores doutores ${ }^{7}$ e 123 grupos de pesquisas registrados no Diretório de Grupos de Pesquisas do Conselho Nacional de Desenvolvimento Científico e Tecnológico $(\mathrm{CNPq})^{8}$, que atuam na área relacionada com ensaios clínicos. As parcerias entre 
a indústria farmacêutica, a academia e o governo, no atual cenário, podem ser benéficas para todas as partes envolvidas, desde que respeitados os limites éticos e os créditos das colaborações $^{4,9}$.

Na tentativa de fortalecer estruturas e grupos de pesquisa clínica no Brasil, vislumbravase, em longo prazo, maior atenção às demandas do Sistema Único de Saúde (SUS), com a redução da dependência estrangeira ${ }^{10}$ e em resposta à Constituição Federal, a qual estabelece em seu artigo 200, inciso $\mathrm{V}^{11}$, entre as competências do Sistema Único de Saúde (SUS), 'incrementar, em sua área de atuação, o desenvolvimento científico e tecnológico e a inovação $^{11,12}$. Nesse sentido, no ano de 2005 e por iniciativa conjunta dos Ministérios da Saúde (MS) e da Ciência Tecnologia, Inovações e Comunicações (MCTIC), e com o apoio deliberativo de representantes de diversas instituições ligadas ao Ensino, Pesquisa e Gestão de Ciência, Tecnologia e Inovação (CT\&I), o governo federal estruturou 18 Unidades de Pesquisas Clínicas (UPC) em hospitais de ensino ${ }^{13,14}$ visando a estabelecer uma redebrasileira de ensaios clínicos ${ }^{12}$ então denominada Rede Nacional de Pesquisa Clínica em Hospitais de Ensino (RNPC).

Respondendo a apelos de diversas instituições, declaradas com capacidade para execução de pesquisas clínicas, no ano de 2009 foram incluídos mais 12 UPCs à RNPC que passou a ser estruturada com 32 UPCs dispostas em 14 estados das cinco regiões ${ }^{15}$. A RNPC conta, desde a sua formação, com um Comitê Gestor, composto pelo MS, MCTIC, Financiadora de Estudos e Projetos (Finep) e o CNPq, além de um Centro Coordenador que corresponde a uma das UPCs e tem como função operar como articulador entre o Comitê Gestor e todas as UPCs. O Centro Coordenador dispõe do suporte de quatro grupos de trabalho: capacitação, regulação, institucionalização e novas moléculas ${ }^{12}$.

A RNPC possui uma estrutura de gestão montada e estabelecida por meio de Portaria Ministerial $^{16}$. A literatura ${ }^{17,18,}$ entretanto, enfatiza que a gestão de redes equivale a uma nova abordagem, em contínua mutação, tendo em vista o surgimento de modelos e arranjos colaborativos, caracterizados, em geral, tanto pela ênfase na cooperação entre atores como na produção de inovação.

Atualmente, estudos e análises sobre as características das estruturas de redes de pesquisa clínica que possam subsidiar a sua gestão foram tratados na literatura brasileira somente a partir da abordagem da gestão de redes sociais ${ }^{19}$. Há, porém, exemplos bemsucedidos, a exemplo do caso da The European Clinical Research Infrastructures Network (ECRIN), iniciativa que corresponde a um consórcio de redes de vários países europeus, 
incluindo a França, Alemanha, Itália, Roma, Espanha, Reino, Irlanda, Suécia, Dinamarca, Finlândia, Suíça, Áustria, Bélgica, Hungria e Polônia ${ }^{20,21}$.

Com o propósito de questionar e, consequentemente, buscar respostas a respeito da capacidade operacional dessa rede para os pesquisadores e gestores da saúde e da ciência e tecnologia no contexto do Brasil, o presente trabalho elenca quais são as características da estrutura da RNPC que dificultam ou promovem esforços de pesquisas clínicas bem- sucedidas, explorando a hipótese de que as dimensões estruturais relacionadas aos recursos humanos, especialidades, fomento, estratégia de gestão, e desenvolvimento institucional são decisivas na estrutura de uma rede de pesquisas para a execução de estudos bem sucedidos.

A importância desta pesquisa recai na possibilidade de análise descritiva das alternativas de conexões relacionais e janelas de oportunidades passíveis de fomentar, expandir e fortalecer a pesquisa clínica junto aos grupos brasileiros.

\section{PROCEDIMENTOS E MÉTODOS}

Trata-se de um estudo exploratório descritivo, com abordagem qualiquantitativa do tipo estudo de caso. Realizou-se pesquisa documental em artigos publicados sobre gestão de rede 19, 22, 23,24, 25,26 , modelos de gestão de redes de pesquisas clínicas ${ }^{20,21,27}$, avaliando-se ainda todos os documentos referentes à RNPC que integram o acervo do Departamento de Ciência e Tecnologia (Decit) da Secretaria de Ciência, Tecnologia e Insumos Estratégicos (SCTIE) do Ministério da Saúde (MS). Este estudo conta com a aprovação do Comitê de Ética em Pesquisas da Faculdade de Medicina da USP. Foram encaminhados, por e-mail, aos 32 coordenadores de UPC da RNPC questionários, com 22 questões, estruturadas em quatro blocos de perguntas pontuais relacionadas à forma de colaboração/interação entre as UPCs, capital social interno e externo à rede, capacidade técnica, científica e desenvolvimento institucional. Os participantes assinaram os Termos de Consentimento Livre e Esclarecido (TCLE). Os dados foram analisados de modo a assegurar o sigilo.

\section{Análise dos dados}

Essa investigação se valeu do método de análise de redes sociais por buscar a compreensão dos aspectos relacionais e estatísticos causais dos fenômenos identificados, semelhantes ao descrito por Rossoni $(2008)^{28}$. Para essa finalidade, foram incluídas, informações associadas ao relacionamento entre as UPCs e os seus integrantes. 
Procedeu-se à identificação e representação da rede de colaborações sob a forma de um grafo a partir de um software desenvolvido especialmente para este estudo. Cada nodo representa uma UPC ou área médica. Determinada aresta ou ligação ilustra as relações entre dois nodos. A rede considerada neste trabalho é não direcionada, isto é, não há diferença na direção do relacionamento entre dois nodos.

A estrutura criada na forma de redes de colaboração permite a utilização de um arcabouço matemático robusto relacionado com a análise de redes sociais. Desse modo, é possível estudar as redes considerando-se as métricas topológicas no sentido de aportar conhecimento e informações de caminhos e entidades mais importantes, e que seria difícil obter a partir da tabulação dos dados. As métricas consideradas são: Centralidade de grau, Centralidade de intermediação, Centralidade de proximidade e Coeficiente de agrupamento.

A visualização da rede foi realizada por meio do software Gephi, utilizando-se o algoritmo de desenho (layout) Force Atlas 2, permitindo a visualização e agrupamento de nodos com maior relacionamento. Dessa forma, nodos com menor relacionamento passam a se posicionar a uma maior distância na visualização.

\section{RESULTADOS E DISCUSSÃO}

Dos 32 coordenadores de UPCs integrantes da Rede convidados a participar do estudo, apenas oito se negaram. Desse modo, algumas informações não correspondem ao universo total da Rede, embora reconheçamos ser confiável e representativa a investigação. Outra limitação se refere à validade externa, característica do método qualitativo. Isto é, os resultados e considerações valem apenas para a rede estudada, e não podem ser estendidas automaticamente a outras redes de pesquisa. Discutem-se nesse estudo os relatos adquiridos a partir dos questionários recebidos e os dados coletados de documentos disponibilizados pelo Decit, amparados pelo suporte da literatura 19, 22, 23, 24, 25,26

Tabela 1 - Distribuição das Unidades de Pesquisa Clínicas integrantes da Rede Nacional de Pesquisa Clínica ampliada, segundo Instituição, Cidade e Região a que pertencem.

\begin{tabular}{|lllc|}
\hline \multicolumn{1}{|c}{ Hospital } & Instituição & Cidade & UF \\
\hline Hospital das Clínicas da Universidade Federal de Goiás (HC-UFG) & UFG & Goiânia. & CO \\
\hline Hospital Universitário de Brasília (HUB - UnB) & UnB & Brasília & CO \\
\hline Fundação de Medicina Tropical do Amazonas (FMTA) & FMTA & Manaus & $\mathrm{N}$ \\
\hline Hospital Universitário João de Barros Barreto (HU-HJBB) & UFPA & Belém & $\mathrm{N}$ \\
\hline
\end{tabular}




\begin{tabular}{|c|c|c|c|}
\hline Hospital Geral de Fortaleza (HGF) & UEC & Fortaleza & $\mathrm{NE}$ \\
\hline Hospital Messejana (HM-UFC) & UFC & Fortaleza & $\mathrm{NE}$ \\
\hline Hospital São Rafael - Monte Tabor (HSR) & HSR & Salvador & $\mathrm{NE}$ \\
\hline Hospital Universitário da Universidade Federal do Maranhão (HU-UFMA) & UFMA & São Luís & $\mathrm{NE}$ \\
\hline Hospital Universitário Onofre Lopes (HU-UFRN) & UFRN & Natal & $\mathrm{NE}$ \\
\hline Hospital Geral da Universidade de Pernambuco (HG-UPE) & UPE & Recife & $\mathrm{NE}$ \\
\hline Hospital Universitário Professor Edgard Santos (HU-UFBA) & UFBA & Salvador & $\mathrm{NE}$ \\
\hline Hospital Universitário Walter Cantídio (HU-UFC & UFC & Fortaleza & $\mathrm{NE}$ \\
\hline Instituto Materno Infantil Professor Fernando Figueira (IMIP) & IMIP & Recife & $\mathrm{NE}$ \\
\hline Hospital de Clínicas de Porto Alegre (HCPA) & UFRGS & Porto Alegre & $\mathrm{S}$ \\
\hline Hospital Nossa Senhora da Conceição (HU-UNISUL) & UNISUL & Florianópolis & $\mathrm{S}$ \\
\hline Hospital São Lucas (PUC/RS) & PUC/RGS & Porto Alegre & $\mathrm{S}$ \\
\hline Hospital Universitário Regional de Maringá (HU-UEM) & UEM & Maringá & $\mathrm{S}$ \\
\hline Instituto de Cardiologia do Rio Grande do Sul (IC-FUC/RS) & FUC & Porto Alegre & $\mathrm{S}$ \\
\hline Fundação Oswaldo Cruz (FIOCRUZ) & FIOCRUZ & Rio de Janeiro & SE \\
\hline Hospital das Clínicas da Faculdade de Medicina de Botucatu (HC-UNESP) & UNESP & Botucatu & SE \\
\hline Hospital das Clínicas da Faculdade de Medicina de Ribeirão Preto (HC-USP/RP) & USP/RP & Ribeirão Preto & SE \\
\hline Hospital das Clínicas da Universidade Estadual de Campinas (HC-UNICAMP) & UNICAMP & Campinas & SE \\
\hline Hospital das Clínicas da Universidade Federal de Minas Gerais (HC-UFMG & UFMG & B. Horizonte & SE \\
\hline Hospital de Clínicas da Faculdade de Medicina da USP (HC-USP) & USP & São Paulo & SE \\
\hline Hospital São Paulo (HU-UNIFESP) & UNIFESP & São Paulo & SE \\
\hline Hospital Universitário Antônio Pedro (HUAP-UFF) & UFF & Niterói & SE \\
\hline Hospital Universitário Clementino Fraga Filho (HU-UFRJ) & UFRJ & Rio de Janeiro & SE \\
\hline Hospital Universitário da Universidade de São Paulo (HU-USP) & USP & São Paulo & SE \\
\hline Instituto de Medicina Integral Professor Fernandes Figueira (FIOCRUZ) & FIOCRUZ & Rio de Janeiro & SE \\
\hline Instituto Nacional de Câncer (INCA) & INCA & Rio de Janeiro & SE \\
\hline Instituto Nacional de Cardiologia Laranjeiras (INC) & UFRJ & Rio de Janeiro & $\mathrm{SE}$ \\
\hline Instituto Nacional de Traumato-Ortopedia (INTO) & INTO & Rio de Janeiro & SE \\
\hline
\end{tabular}

Fonte: Elaboração própria com adaptações de Brasil (2010) ${ }^{12}$

Diferentemente das demais UPCs (Tabela 1) que se localizam em ambientes de enfermarias de hospitais universitários, a UPC da Fundação Oswaldo Cruz, funciona como um Academic Research Organization (ARO), ou seja, um escritório gerencial que acompanha os 370 estudos que estão sendo executados por aproximadamente 60 grupos de pesquisas dispersos nas seguintes unidades: Instituto Leônides e Maria Deane/AM, Centro de Pesquisas René Rachou (CPqRR)/MG, Centro de Pesquisas Ageu Magalhães (CPqAM)/PE, Instituto Gonçalo Moniz (IGM)/BA, Instituto Carlos Chagas (ICC)/PR, Instituto de Tecnologia em Fármacos (Farmanguinhos)/RJ, Instituto de Tecnologia em Imunobiológicos (Biomanguinhos)/RJ, Instituto Nacional de Infectologia Evandro Chagas (INI)/RJ, Instituto Nacional de Saúde da Mulher, da Criança e do Adolescente Fernandes Figueira (IFF), Instituto Oswaldo Cruz (IOC)/RJ. Particularmente a Fiocruz opera uma rede interna denominada Rede Fiocruz de Pesquisa Clínica, sendo essa integrante da RNPC e, ao mesmo tempo, independente. 


\section{Recursos Humanos na Rede}

O processo estratégico para o fortalecimento do Complexo Industrial e Econômico da Saúde (CEIS) ${ }^{29,30}$ - materializado, a partir da instalação das UPCs em hospitais de ensino, vem favorecendo a capacitação de recursos humanos da área médica quanto aos aspectos éticos e regulatórios da pesquisa clínica e indubitavelmente promove a discussão de pesquisas com foco no SUS ${ }^{1,3}$. A partir de 2010 foram fomentados, no âmbito da RNPC, cursos de pesquisa clínica nas modalidades básica, avançada e de pós-graduação (especialização), além de treinamentos diversos.

Excluindo-se os pesquisadores da Fiocruz, pelos motivos já mencionados neste artigo, a RNPC conta, atualmente, com aproximadamente 327 profissionais colaboradores, dos quais $75 \%$ possuem formação específica em gestão e execução de pesquisa clínica e $72 \%$ têm formação em nível de mestrado e doutorado - todos atuando na área. 100\% das unidades dispõem de profissionais médicos, enfermeiros e farmacêuticos. Algumas UPCs têm em seus quadros, adicionalmente, biólogos, nutricionistas, fisioterapeutas, dentistas, epidemiologistas, estatísticos administradores e educadores físicos.

Na Figura 1 está disposta a representação na forma de Rede de colaboração entre as UPCs. A abordagem suscitada corresponde a colaborações voltadas para a realização de estudos e de intercâmbio técnico e/ou de formação. O destaque para a UPC da UFMG ocorreu, especialmente, em função de essa unidade ter sido o centro coordenador da Rede no período de 2010 a 2014, desenvolvendo a capacitação científica a todas unidades integrantes. Já a unidade HCPA, o primeiro centro coordenador da Rede, identificada como a segunda UPC mais colaborativa na RNPC, não fomentou intensa capacitação científica, como a do HC/UFMG, mas firmou, entretanto, parceria para a realização de estudos multicêntricos em pelo menos 70,4\% das UPCs da Rede.

Esse tipo de representação permite observar e identificar quais as UPCs que se destacam como centrais ou perifericas.

Figura 1 - Colaboração entre as Unidades de Pesquisa Clínica da Rede Nacional de Pesquisas Clínicas em Hospitais de Ensino, no período de 2010 a 2014. 


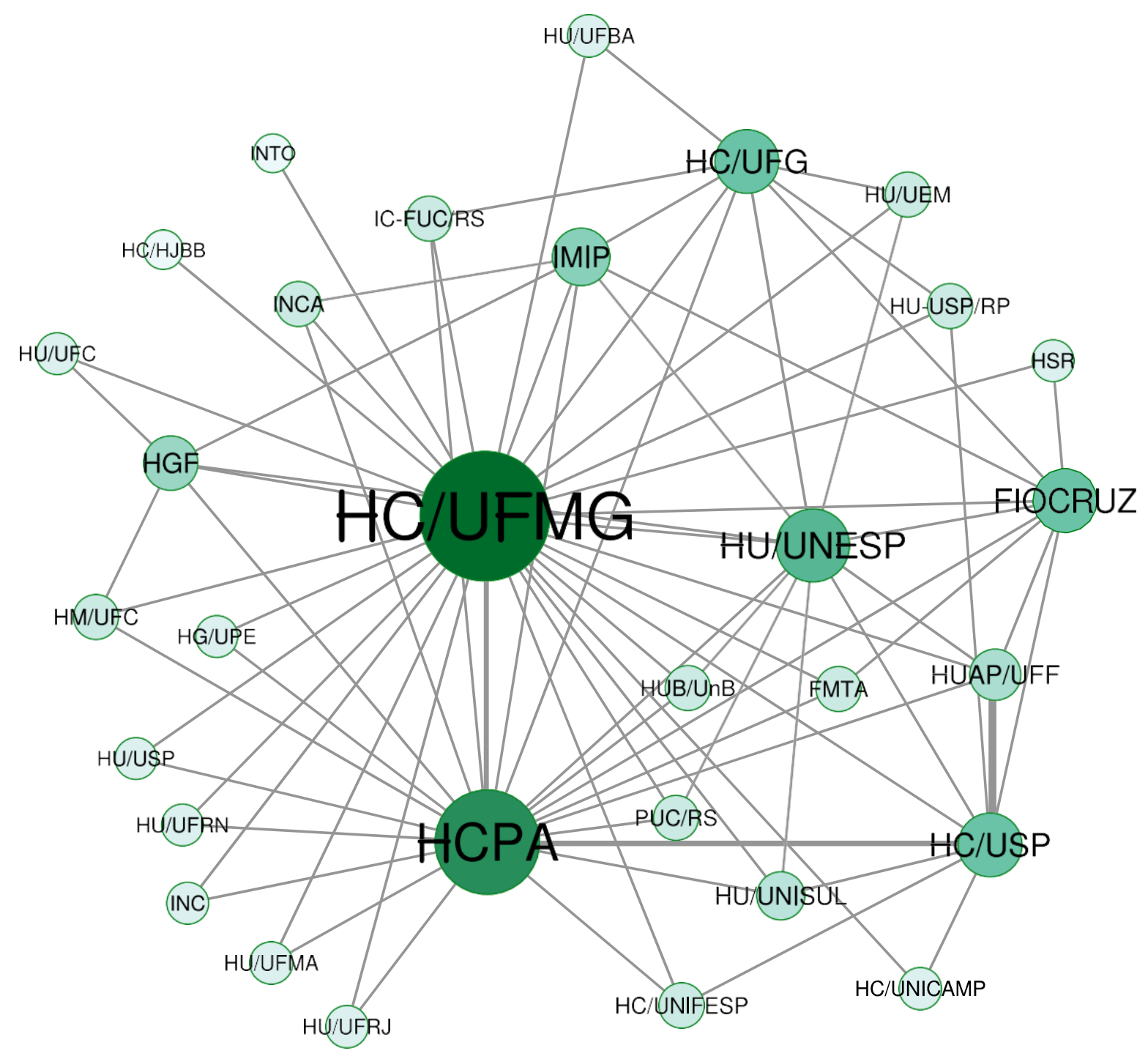

Fonte: Elaboração própria a partir dos questionários aplicados.

\section{Especialidades e Conexões}

É importante destacar que não havia registro algum nos documentos analisados, publicados ou internos, disponibilizados pelo Decit, no que diz respeito à capacidade de articulação entre as UPCs da Rede, seja pela capacidade de execução de estudos por fases dos ensaios clínicos, especialidades seja por qualquer outro tipo de colaboração.

Foram identificadas nos questionários 81 especialidades médicas, declaradas pelos representantes das UPCs em suas instituições, conforme apresentadas no Quadro 1 a seguir, para as quais fomos fiéis aos relatos por parte dos respondentes. As especialidades com mais centros disponíveis são, respectivamente: Cardiologia, Reumatologia, Oncologia, Neurologia e Ginecologia. 
Quadro 1 - Especialidades médicas disponíveis nas Unidades de Pesquisas Clínicas, segundo reportado pelos integrantes das UPC da Rede Nacional de Pesquisa Clínica.

Fonte: Elaboração própria a partir dos questionários recebidos.

As especialidades médicas apresentadas no Quadro 1 foram compiladas e transformadas em áreas médicas visando à construção de uma rede de relacionamento (veja

\begin{tabular}{|c|c|c|c|}
\hline$\checkmark$ Obesidade mórbida & $\checkmark$ Doenças do olho e anexos & $\checkmark$ Hipercolesterolemia & $\checkmark$ Tuberculose \\
\hline$\checkmark$ Anestesiologia & $\checkmark$ Obstetrícia & $\checkmark \mathrm{HIV}$ & $\checkmark$ Oftalmologia \\
\hline$\checkmark$ Câncer digestivo & $\checkmark$ Hepatites & $\checkmark$ Infecção cirúrgica & $\checkmark$ Endocrinologia \\
\hline$\checkmark$ Pneumologia & $\checkmark$ Doenças infecciosas & $\checkmark$ Infectologia & $\checkmark$ Enxaqueca \\
\hline$\checkmark$ Proctologia & $\checkmark$ Parkinson & $\checkmark$ Dengue & $\checkmark$ Urologia \\
\hline$\checkmark$ Cirurgia geral & $\checkmark$ Reumatologia & $\checkmark$ Dermatologia & $\checkmark$ Pediatria \\
\hline$\checkmark$ Tireoidopatias & $\checkmark$ Oncologia & $\checkmark$ LER & $\checkmark$ Cardiologia \\
\hline$\checkmark$ Cirurgia cardíovascular & $\checkmark$ Ortopedia & $\checkmark$ Lúpus & $\checkmark$ Cérebro-vascular \\
\hline$\checkmark$ Terapia intensiva & $\checkmark$ Epilepsia & $\checkmark$ Anestesiologia & $\checkmark$ Psoríase \\
\hline$\checkmark$ Clínica médica & $\checkmark$ Odontologia & $\checkmark$ Medicina Tropical & $\checkmark$ Retinopatia \\
\hline$\checkmark$ Demências & $\checkmark$ Esclerose múltipla & $\checkmark$ Traumatologia & $\checkmark$ Doenças renais \\
\hline$\checkmark$ Neoplasmas & $\checkmark$ Fibrilação arterial & $\checkmark$ Nefrologia & $\checkmark$ Cirurgias \\
\hline$\checkmark$ Insuficiência renal & $\checkmark$ Gastroenterologia & $\checkmark$ Insuficiência cardíaca & $\checkmark$ Cirurgia cardíaca \\
\hline$\checkmark$ Diabetes & $\checkmark$ Ginecologia & $\checkmark$ Neurologia & $\checkmark$ Vacinas \\
\hline$\checkmark$ Hepatologia & $\checkmark$ Doenças & $\checkmark$ Nutrição & $\checkmark$ Transplante renal \\
\hline$\checkmark$ Doença de chagas & neuroinfecciosas & $\checkmark$ Malária & $\checkmark$ Hematologia \\
\hline $\begin{array}{l}\checkmark \text { Afecções originadas no } \\
\text { período perinatal }\end{array}$ & $\begin{array}{l}\checkmark \text { Doenças do aparelho } \\
\text { respiratório }\end{array}$ & $\begin{array}{l}\checkmark \text { Doenças do aparelho } \\
\text { geniturinário }\end{array}$ & $\begin{array}{l}\checkmark \text { Esclerose lateral } \\
\text { amiotrófica }\end{array}$ \\
\hline $\begin{array}{l}\checkmark \text { Micobactéria não } \\
\text { tuberculosa }\end{array}$ & $\begin{array}{l}\checkmark \text { Síndrome coronariana } \\
\text { aguda }\end{array}$ & $\begin{array}{l}\checkmark \text { Transtornos mentais e } \\
\text { comportamentais }\end{array}$ & $\begin{array}{l}\checkmark \text { Doenças do aparelho } \\
\text { digestivo }\end{array}$ \\
\hline $\begin{array}{l}\checkmark \text { Doenças do aparelho } \\
\text { circulatório }\end{array}$ & $\begin{array}{l}\checkmark \text { Doenças do sistema } \\
\text { nervoso }\end{array}$ & $\begin{array}{l}\checkmark \text { Doenças infecciosas e } \\
\text { parasitárias }\end{array}$ & $\begin{array}{l}\checkmark \text { Doenças } \\
\text { cardiovasculares }\end{array}$ \\
\hline \multicolumn{2}{|c|}{$\checkmark$ Doenças endócrinas, nutricionais e metabólicas } & \multicolumn{2}{|c|}{$\checkmark$ Doenças do sistema osteomuscular e do tecido conjuntivo } \\
\hline \multicolumn{2}{|c|}{$\begin{array}{l}\checkmark \text { Malformações congênitas, deformidades e anomalias } \\
\text { cromossômicas }\end{array}$} & \multicolumn{2}{|c|}{$\begin{array}{l}\checkmark \text { Sintomas, sinais e achados anormais de exames clínicos e } \\
\text { de laboratório não classificados em outra parte }\end{array}$} \\
\hline \multicolumn{2}{|c|}{$\begin{array}{l}\checkmark \text { Doenças do sangue e dos órgãos hematopoiéticos e alguns } \\
\text { transtornos imunitários }\end{array}$} & \multicolumn{2}{|c|}{$\begin{array}{l}\checkmark \text { Lesões, envenenamentos e algumas outras consequências } \\
\text { de causas externas }\end{array}$} \\
\hline
\end{tabular}

Figura 2). A partir dela é possível identificar a capacidade de interação entre as UPCs, demonstrando as possibilidades de estabelecimento de parcerias para a execução de atividades, projetos ou estudos. Nesse sentido, a Rede configura uma grande janela de oportunidades, conforme defendido por Viana et al. $(2011)^{29}$, ao se referir, nesse contexto, ao papel central do Ministério da Saúde enquanto membro gestor da RNPC.

Ainda sobre a janela de oportunidades, Guimarães (2011) ${ }^{1}$ afirma que a competitividade internacional na geração de inovação tem-se voltado à compreensão de como se desenvolvem e se articulam os atores nesse processo, objetivando justamente qualificar tais intervenções para se valer dos processos estratégicos em favor do fortalecimento do CEIS. 
Figura 2 - Sociograma das áreas médicas disponíveis nas Unidades de Pesquisas Clínicas segundo relatos dos questionários.

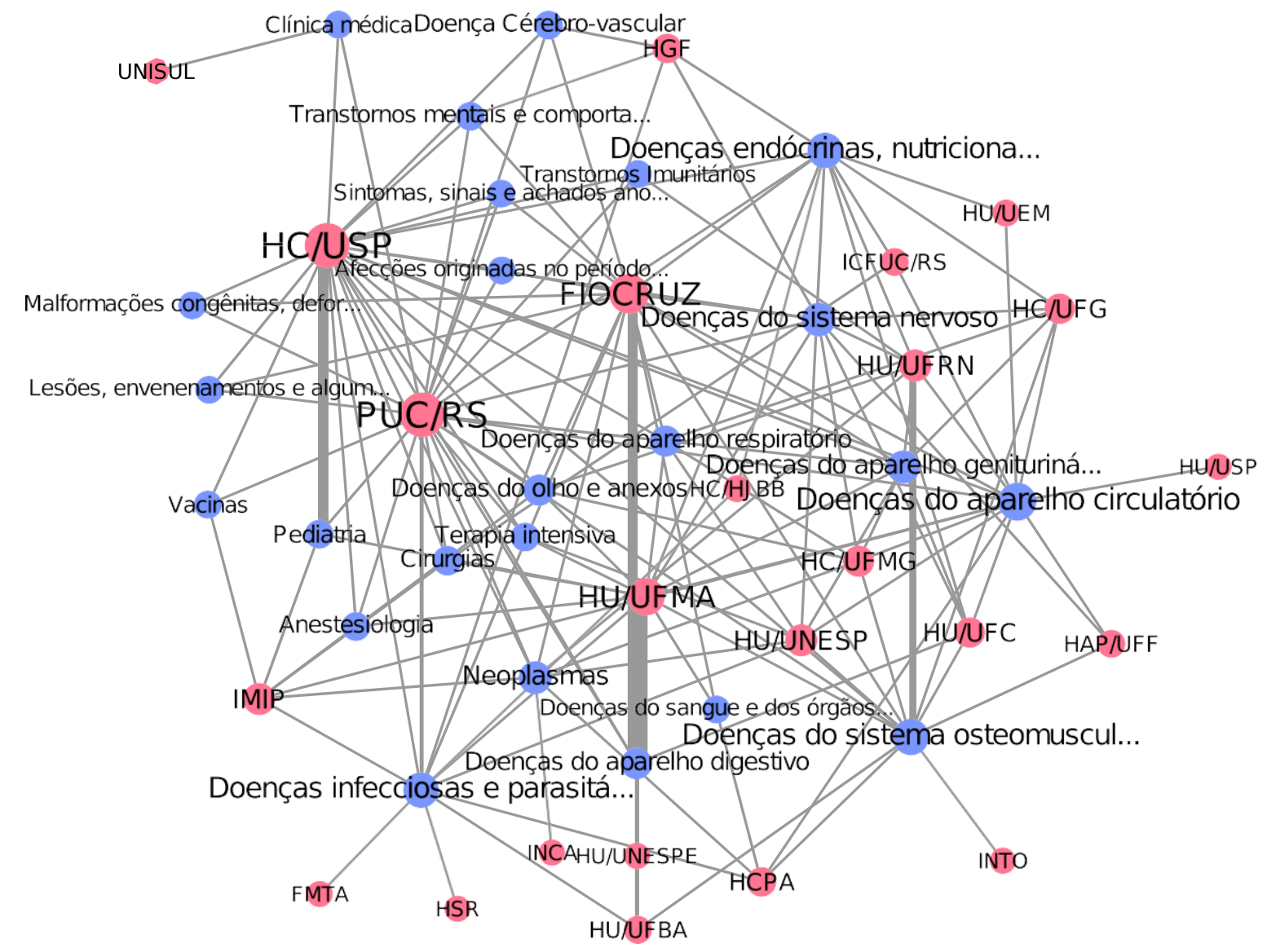

Fonte: Elaboração própria a partir dos questionários aplicados.

Legenda: Enquanto cada nodo escuro representa uma UPC, o nodo claro ilustra uma área médica, a qual abriga várias especialidades. A ligação entre eles significa que tais UPCs possuem capacidade técnica-científica para desenvolver estudos na área. Os nodos foram dimensionados de acordo com o número de conexões possíveis entre as UPCs e as áreas médicas.

A partir do estabelecimento dessas conexões (Figura 2) é possível viabilizar a criação de um portfólio voltado às competências da Rede.

\section{O fomento à Pesquisa para a Rede}

Após a formação da Rede com investimentos na ordem de 35 milhões de reais para estruturação das UPCs, foram lançadas cinco chamadas públicas para fomentar a execução de 
estudos na RNPC nos anos de 2007, 2008, 2010 e duas em 2013, visando a fortalecer a Rede e formalizar parcerias entre as UPCs. O Decit/MS em parceria com os Fundos Setoriais de Saúde (CT-Saúde) do MCTIC aportaram R \$ 68 milhões de reais para o conjunto de chamadas. Desse volume de recursos, entretanto, foram empenhados apenas $\mathrm{R} \$ 54,3$ milhões de reais para contratação de 55 projetos de pesquisas, tendo em vista vários fatores, incluído principalmente, dificuldades com os tramites documentais dos contemplados junto às suas fundações institucionais. $77 \%$ dos projetos contratados eram provenientes de instituições componentes da RNPC, entretanto, a maior parte delas não tinha colaboração com as UPCs.

A distribuição de recursos e projetos de pesquisa se concentrou, sobretudo, nos estados do Rio Grande do Sul (29\% dos recursos e 16\% dos projetos), São Paulo (28\% dos recursos e $29 \%$ dos projetos), Rio de Janeiro ( $22 \%$ dos recursos e $15 \%$ dos projetos). Chama atenção a região Norte se posicionar a frente da região Centro Oeste em captação de recursos e projetos de grande porte, ao que se considerou a expertise desenvolvida na Fundação de Medicina Tropical do Amazonas voltada ao tratamento das doenças negligenciadas, especialmente a malária.

Tabela 2 - Distribuição dos recursos e número de projetos por percentual, segundo as regiões brasileiras - Editais 2007, 2008, 2010 e 2013.

\begin{tabular}{ccccc}
\hline Região & N. Projetos & \% projetos & R\$ recebidos & \% de recursos \\
\hline CO & 4 & $7 \%$ & 1.16 milhões & $2 \%$ \\
N & 2 & $4 \%$ & 1.25 milhões & $3 \%$ \\
NE & 9 & $16 \%$ & 5.41 milhões & $10 \%$ \\
S & 14 & $26 \%$ & 18.62 milhões & $34 \%$ \\
SE & 26 & $47 \%$ & 27.84 milhões & $51 \%$ \\
\hline
\end{tabular}

Fonte: Elaboração própria a partir de documentos internos do DECIT.

A partir das informações constantes na Tabela 2, é possível reafirmar a concentração historicamente marcada pela maior densidade de capacidade técnica, científica e de infraestrutura das regiões sudeste e sul do país.

Quanto às instituições que receberam maior volume de recursos, foram respectivamente: Hospital de Clínicas de Porto Alegre- HCPA, Universidade Estadual Paulista - UNESP e Universidade de São Paulo - USP, Pontifícia Universidade Católica do Rio Grande do Sul - PUC/RS e o Instituto do Câncer - INCA.

Varias questões corroboraram para que o esforço em fomentar parcerias no âmbito da 
RNPC, por meio de chamadas públicas, não fosse atingido: 1) não exigiam que os projetos fossem multicêntricos nem construídos em parcerias com as UPCs membros da Rede; 2) os critérios de elegibilidade não explicitavam que a Rede estava restrita às UPCs, possibilitando que outras UPCs das instituições fossem beneficiadas, 3) não eram exclusivos para ensaios clínicos; e 4) não vetavam a submissão de estudos observacionais.

\section{Estratégia de Gestão}

A RNPC possui mecanismo de gestão verticalizado, que se utiliza de um comitê gestor, um centro coordenador e quatro grupos de trabalho. Possui estratégia de comunicação, que consiste em encontros mensais por meio de um espaço virtual situado no Centro de Apoio de Educação à Distância (CAED) da UFMG, com o suporte técnico da Rede Universitária de Telemedicina (Rede-RUTE), a qual tem como objetivo o incentivo de trabalhos multicêntricos. As reuniões compõem vias de mão dupla por constituírem um espaço de compartilhamento de informações do Comitê Gestor para as UPCs e vice-versa. No entanto, segundo o nosso entendimento, a adesão das UPCs às reuniões é mínima. Ao longo de um ano, a reunião com maior número de unidades participantes contou com 12 representantes.

Faz-se importante destacar que a diversidade de instituições que compõem uma rede exige uma gestão horizontalizada com fluxo de conhecimento sem hierarquias e que aposte no gerenciamento colaborativo ${ }^{31}$, no qual os indivíduos sejam capazes de exercer sua criatividade e colocar em prática novas ideias. Sem dúvida, esse é o grande desafio da gestão de inovação.

Nesse sentido instituiu-se um grupo de trabalho por meio da portaria ministerial $n^{\circ} 4$ de 12 de março de $2015^{32}$, composto por representantes do MS que o coordena, MCTIC e Anvisa, com as atribuições de propor novo modelo de gestão para a RNPC, elaborar proposta de regimento interno e de diretrizes para o trabalho em rede, efetivamente.

A necessidade e a tentativa de incrementar a RNPC são latentes, entretanto se esbarrou em questões gerenciais internas ao Decit/SCTIE/MS, que correspondeu à substituição de três gestores à frente da Secretaria, ocasionando o estabelecimento de diferentes fluxos de trabalho, se perdendo, portanto, muito dos trabalhos em andamento.

\section{Desenvolvimento Institucional}

Segundo relatos dos questionários, a RNPC vem imprimindo capital social aos seus 
integrantes no âmbito institucional (45\%) e um pouco menos (40\%) fora dela. Atualmente estão sendo desenvolvidos 722 projetos nas instituições integrantes da Rede com a participação das UPCs. Apenas $15 \%$ delas, porém, confirmaram que os projetos de pesquisa clínica da instituição estão obrigatoriamente sob o monitoramento da UPC. Por outro lado, quatro unidades revelaram não se relacionar com os projetos realizados na instituição, e outras três UPCs não detinham a informação, sugerindo, assim, falta de entrosamento no âmbito institucional.

Não há registro fidedigno em todas as UPCs sobre o número de pós-graduandos que já utilizaram as instalações para execução de seus projetos acadêmicos. Entretanto, foram identificados 1.017 alunos em nível de mestrado e doutorado, seja para capacitação em técnicas da pesquisa clínica seja para a utilização de equipamentos das unidades, desde o período que a UPC integrou à RNPC (2005 ou 2009) até dezembro de 2015. De acordo com Guimarães $(2011)^{2}$, o papel da política de pós-graduação no setor de saúde humana nos próximos anos deveria ser o de reforçar o sistema setorial de inovação em saúde, enfrentando o desafio de articular de maneira sólida a pós-graduação com as prioridades do SUS (prioridades de pesquisa e de serviço). Segundo Maia $(2004)^{33}$, na medida em que se entenda rede, em seu conceito ampliado, é necessário desenvolver novos conceitos para gerenciamento das redes organizacionais. Nesse caso, reforçar o papel da RNPC no âmbito institucional é absolutamente estratégico.

\section{CONSIDERAÇÕES FINAIS}

A partir desta análise, é possível inferir que as estruturas que promovem resultados bem-sucedidos no âmbito da RNPC estão relacionadas à capacitação de profissionais, ao fomento à pesquisa com foco no SUS em ambiente acadêmico de pós-graduação e ao intercâmbio técnico científico entre as instituições. Os resultados sinalizam que tanto as expertises quanto as forças institucionais podem contribuir para a eficácia das colaborações, devendo ser explorada e fortemente incentivada entre os integrantes da Rede.

Com relação às estruturas que dificultam resultados bem-sucedidos, considera-se que estas padecem de falhas de gestão. Foi verificada a ausência de acompanhamento e avaliação das UPCs, bem como de detenção de conhecimento sobre as estruturas e capacidades técnicas operacionais de cada UPC, por parte do comitê gestor. O relacionamento entre as UPCs também mostrou fragilidades e pouca interação. Foi identificada a necessidade de se introduzir 
na rotina da Rede uma abordagem em que se contemple o monitoramento do desempenho atual e, simultaneamente, focalize a atuação futura, buscando, desse modo, potencializar energias, lideranças e expertises dos integrantes, em prol da Rede.

Como principal dificuldade para que a RNPC se mantenha aquecida, identificamos a ausência de geração de demandas pelo Comitê Gestor, as quais reflitam as prioridades da saúde pública brasileira, viabilizando a criação de um portfólio voltado às competências da Rede. Esse foi um dos principais entraves encontrado na estrutura da RNPC, em comparação às redes bem-sucedidas, a exemplo das redes componentes da The European Clinical Research Infrastructures Network (ECRIN $)^{21}$.

Destacamos ainda a necessidade da criação de uma home page da RNPC, que seja funcional e interativa aos participantes e, ao mesmo tempo, confira visibilidade externa para a Rede.

Acreditamos que este estudo possa constituir um significativo insumo para a elaboração de um plano estratégico de reposicionamento das instituições integrantes da RNPC, no sentido de ampliar e validar ainda mais as suas conexões, a fim de expandir as colaborações além das fronteiras, legitimando, assim, mesmo que em longo prazo, a pesquisa clínica com foco no SUS em território brasileiro. 


\section{REFERÊNCIAS}

1. Pinho RG, Paiva VP, Borges AL, Paulo JP. Ensaios clínicos no Brasil: competitividade internacional e desafios. Complexo Industrial da Saúde BNDES Setorial 2012; 36: 45-84. [Internet] Disponível em:

http://www.bndes.gov.br/SiteBNDES/export/sites/default/bndes_pt/Galerias/Arquivos/conhe cimento/bnset/set3602.pdf (Acessado em 15/12/2015).

2. Guimarães R. Desafios da pós-graduação em saúde humana no Brasil. Rev. Saúde Pública 2011; 45(1): 1-13.

3. Caramori CA. Institucionalização da Pesquisa Clínica na Faculdade de Medicina de Botucatu UNESP: Pesquisa Clínica, Ensino Médico, Desenvolvimento, Inovação 2013; [Internet] Disponível em: https://books.google.com.br/books/about/Institucionaliza\%C3\%A7\%C3\%A3o_da_Pesquisa_ $\mathrm{Cl} \% \mathrm{C} 3 \%$ ADni.html?hl=pt-BR\&id=K2dbBAAAQBAJ (Acessado em 13/12/2015).

4. Dainesi SM, Goldbaum M. Pesquisa clínica como estratégia de desenvolvimento em saúde. Revista da Associação Médica Brasileira 2012; 58(1): 2-6.

5. Ministério da Saúde. Conselho Nacional de Saúde. Resolução No 196 de 10 de outubro de 1996; [Internet] Disponível em: http://conselho.saude.gov.br/comissao/conep/resolucao.html (Acessado em 12/12/2015).

6. Resolução Nº 466 de 12 de dezembro de 2012; [Internet] Disponível em: http://conselho.saude.gov.br/resolucoes/2012/Reso466.pdf (Acessado em 12/12/2015).

7. Conselho Nacional de Ciência e Tecnologia (CNPq). Plataforma Lattes. [Internet] Disponível em: http://buscatextual.cnpq.br/buscatextual/busca.do (Acessado em 04/06/2016).

8. D__ Diretório dos Grupos de Pesquisas. [Internet] Disponível em: http://dgp.cnpq.br/dgp/faces/consulta/consulta_parametrizada.jsf (Acessado em 04/06/2016).

9. Zago MA. A pesquisa clínica no Brasil. Ciência \& Saúde Coletiva 2004; 9(2): 363-374.

10. Paula AP, Giozza SP, Pereira MZ, Boaventura PS, Santos LMP, Sachetti CG, et al. Clinical investigations for SUS, the brazilian public health system. São Paulo Med J. 2012; 130(3): 179-86.

11. Brasil. Constituição da República Federativa do Brasil 1988; [Internet] Disponível em: http://www.planalto.gov.br/ccivil_03/constituicao/ConstituicaoCompilado.htm (Acessado em 10/12/2015). 
12. Ministério da Saúde. Rede Nacional de Pesquisa Clínica no Brasil: Resposta e redução da dependência estrangeira. Rev. Saúde Pública 2010; 44(3): 575-8. [Internet] Disponível em: http://www.scielosp.org/pdf/rsp/v44n3/24.pdf (Acessado em 10/12/2015).

13. Financiadora de Estudos e Projetos. Chamada Pública MCT/MS/FINEP - Ação Transversal - Implantação de Unidades de Pesquisa Clínica 04/2005; [Internet] Disponível em: http://www.finep.gov.br/chamadas-publicas/chamadapublica/481 (Acessado em 13/12/2015).

14. Ministério da Saúde. Relatório de Gestão 2006 do Departamento de Ciência e Tecnologia em Saúde (DECIT) 2007; 268 p. [Internet] Disponível em:

http://bvsms.saude.gov.br/bvs/publicacoes/relatorio_gestao_decit_2006.pdf 2 (Acessado em $13 / 12 / 2015)$.

15.

Proposta para ampliação da Rede Nacional de Pesquisa Clínica. Brasília 2009;

[Internet] Disponível em:

http://www.portal.saude.gov.br/portal/arquivos/pdf/ampliação_RNPC.pdf (Acessado em $12 / 12 / 2015)$

16. ___ Portaria $\mathrm{N}^{\circ} 137$, de 24 de janeiro de 2014. Dispõe sobre as Redes Nacionais de Pesquisa em Saúde. [Internet] Disponível em:

http://bvsms.saude.gov.br/bvs/saudelegis/gm/2014/prt0137_24_01_2014.html (Acessado em 05/01/2016).

17. Nodari CH, Camargo ME, Olea PM, Dorion ECH, Claus SM. Configuração das práticas de inovação na atenção primária à saúde: estudo de caso. Ciência \& Saúde Coletiva 2015; 20 : 3073-3086.

18. Vale GMV, Amâncio R, Lima JB. Criação e Gestão de Redes na Estratégia Competitiva de Empresas e Regiões. RAUSP. Revista de Administração 2006; 41(2): 136-146. [Internet] Disponível em: www.rausp.usp.br/download.asp?file=V4102136.pdf (Acessado em $12 / 12 / 2015)$

19. Rodan S, Galunic C. More than network structure: how knowledge heterogeneity influences managerial performance and innovativeness. Strat. Mgmt. J. 2004; 25: 541-562.

20. Demotes-Mainard J, Kubiak C. A European perspective - the European clinical research infrastructures network. Annals of Oncology 2011; 22 (7): vii44-vii49.

21. Kubiak C, Andres-Trelles F, Kuchinke W, Huemer KH, Thirstrup S, et al. Common definition for categories of clinical research: a prerequisite for a survey on regulatory requirements by the European Clinical Research Infrastructures Network (ECRIN). Trials 2009; 10:95. 
22. Varda DM, Retrum JH. An exploratory analysis of network characteristics and quality of interactions among public health collaborative. J Public Health Res. 2012; 1(2): 170-176.

23. Long JC, Cunningham FC, Braithwaite J. Bridges, brokers and boundary spanners in collaborative networks: a systematic review. BMC Health Services Research 2013; 13:158.

24. Corley E, Boardman C, Bozeman B. Design and the management of multi-institutional research collaborations: Theoretical implications from two case studies. Research Policy 2006; 35: 975 - 993.

25. Youtie J, Libaers D, Bozeman B. Institutionalization of university research centers: The case of the National Cooperative Program in Infertility Research. Technovation 2006; 26(9): 1055-1063.

26. Fleury, SMT. Gestão de redes: a estratégia de regionalização da política de saúde. Rio de Janeiro: Editora FGV; 2011.

27. The Clinical Research Network. [Internet] Disponível em:

https://www.crn.nihr.ac.uk/networks/ (Acessado em 14/01/2016).

28. Rossoni L, Hocayen-da-Silva AJ, Ferreira Junior I. Estrutura de Relacionamento entre Instituições de Pesquisa do Campo de Ciência e Tecnologia no Brasil. Rev Adm Emp 2008; 48: $34-48$.

29. Viana ALD, Iozzi FL, Albuquerque MV, Bousquat A. Saúde, desenvolvimento e inovação tecnológica: nova perspectiva de abordagem e de investigação. Lua Nova: Revista de Cultura e Política 2011; 83(1): 41-77.

30. Costa LS, Gadelha CAG, Maldonado J. A perspectiva territorial da inovação em saúde: a necessidade de um novo enfoque. Rev. Saúde Pública 2012; 46(1): 59-67.

31. Martins WJ, Artmann E, Rivera FJU. Gestão comunicativa para redes cooperativas de ciência, tecnologia e inovação em saúde. Rev. de Saúde Pública 2012; 46(spe), 51-58.

32. Ministério da Saúde. Portaria No 4 de 12 de março de 2015. Dispõe sobre a instituição de Grupo de Trabalho para definição de diretrizes gerais de operacionalização da Rede Nacional de Pesquisa Clínica (RNPC). [Internet] Disponível em:

http://bvsms.saude.gov.br/bvs/saudelegis/sctie/2015/prt0003_09_03_2013.html (Acesso em 23/06/2016.

33. Maia PRS, Novak FR, Almeida JAG, Silva DA. Bases conceituais para uma estratégia de gestão: o caso da Rede Nacional de Bancos de Leite Humano. Cad. Saúde Pública 2004; 20 (6): 1700-1708. 